\title{
- Sorry to Interrupt, But May I Have Your Attention? Preliminary Design and Evaluation of Autonomous Engagement in HRI
}

\author{
Vincent Rousseau, François Ferland, Dominic Létourneau, François \\ Michaud \\ Department of Electrical Engineering and Computer Engineering, Université de \\ Sherbrooke, Québec, Canada.
}

The design and the evaluation of an autonomous interactive robot is a challenging research endeavor because there is as much to learn from the interaction between the integrated technologies as there is from the embodied human-robot interaction, in addition to observing their mutual interdependencies. This paper reports on IRL-0, a prototyping platform that we used to conduct preliminary studies on the influences of combining verbal and nonverbal modalities (facial expressions, head movement, arm gestures, and approach trajectory) for engaging interaction with people in controlled conditions and in real-world settings. IRL-0 is made of a compliant omnidirectional mobile base equipped with an expressive face and a three degrees-of-freedom (DOFs) compliant arm. By assembling this prototype and conducting these preliminary studies, our objective is to acquire insights in terms of design (e.g., technology, control) and experimental procedures that are important to take into consideration for the designing and evaluating autonomous robots engaging interaction with people.

Keywords: Autonomous interaction, Engagement, Facial expression and Head movement, Arm gestures, Mobility, Integrated design and evaluation methodology

\section{Introduction}

Having a robot autonomously engage interaction with someone involves the integration of multiple modalities such as voice, gesture, and motion. The influences of such integration are commonly seen in human-human interaction. Let us take, for instance, the case of a group of cub scouts selling calendars in a mall during a fundraising activity: it can be observed that the kids who are more expressive and move toward people are more successful in engaging interaction, compared to the ones that remain behind a counter or stand still and seek interest just through vocal requests.

The design and the evaluation of interactive platforms are usually independently conducted research activities, but they also mutually influence each other: the interaction modalities implemented on a platform influence how people respond, and how people interact with an autonomous robot

\footnotetext{
Due to a conflict of interest with the managing editor Michael Goodrich, the editor for this paper was guest editor Magnus Egerstedt. Authors retain copyright and grant the Journal of Human-Robot Interaction right of first publication with the work simultaneously licensed under a Creative Commons Attribution License that allows others to share the work with an acknowledgement of the work's authorship and initial publication in this journal.
}

Journal of Human-Robot Interaction, Vol. 2, No. 3, 2013, Pages 41-61. DOI 10.5898/JHRI.2.3.Rousseau 
may justify specific design requirements for interaction. Recent robots like Rollin' Justin (Borst et al., 2009), PR2 (Wyrobek, Berger, der Loos Van, \& Salisbury, 2008), Cody (Chen \& Kemp, 2011) and Care-O-bot (Graf, Parlitz, \& Hagele, 2009) bring advanced and secure interaction capabilities by providing elasticity in the robots' manipulators and through omnidirectional mobility. However, their design was not specifically influenced by the tight coupling of interaction modalities for engaging people. Our research laboratory is currently leading a five-year research initiative involving the design and the evaluation of the autonomous platform,IRL-1 (Ferland et al., 2012) (formerly known as Johnny-0 (Michaud, Ferland, Létourneau, Legault, \& Lauria, 2010)). IRL-1 is a humanoid mobile platform capable of bidirectional interaction with humans in natural ways using motion, language, touch, and affect. It integrates new technologies developed specifically for natural interaction, such as Differential Elastic Actuators (DEAs) (Lauria, Legault, Lavoie, \& Michaud, 2008; Lauria, Michaud, et al., 2008) ffor safe and compliant actuation at the omnidirectional locomotion and manipulation levels, and artificial audition with sound source localization, tracking, and separation (Valin, Michaud, \& Rouat, 2007). One situation that we expect IRL-1 to experience is the necessity to engage someone, whether it is for assistance (e.g., for recharging, grabbing an object out of its reach, or acquiring information) or to fulfill a specific task (e.g., for handing objects or directing people to do specific actions). This involves verbal and nonverbal interaction modalities (eye gaze, facial expression, arm gestures, and mobility) tightly coupled with the physical, perceptual, computational, cognitive, energy, and acting capabilities of the robot, allowing it to adapt to the unpredictability of interacting with humans in real-world settings. IRL-1's targeted contributions are in simultaneously exploiting compliance for omnidirectional locomotion and manipulation, grounding reasoning and actions through behavior-producing modules and collectively considering all possible factors experienced 'in the wildness' of the real world (Michaud, Ferland, et al., 2010).

To deal with the complexities of designing an autonomous interactive robot like IRL- 1, we adopted an incremental design approach in which we first implemented proof-of- concept prototypes of the robot's subsystems, i.e.: a) an omnidirectional and compliant locomotion platform (Ferland et al., 2010); b) an interactive arm using DEAs (Legault et al., 2008); c) an expressive face (Shayganfar, Rich, \& Sidner, 2012); and d) a robot control architecture (Michaud, Ferland, et al., 2010). This allowed us to demonstrate and to characterize the capabilities of each subsystem. We then decided to conduct a preliminary integration using the available proof-of-concept prototypes to create IRL-0 for the following reasons:

- Integration of designed interaction modalities is a key challenge in making an autonomous interactive robot; and

- Human-robot interaction (HRI) brings additional dimensions (e.g., technological capabilities, dynamics of the interaction, environmental constraints, etc.) that can only be observed by having the robot operate in the intended conditions.

IRL-0 was therefore made to conduct preliminary HRI system studies exploring the influences of nonverbal modalities in making an autonomous robot engage people's attention and interaction. Many useful observations can be made by conducting such preliminary studies with working systems in realistic conditions, as we had noted in our earlier works on child-robot interaction (Michaud et al., 2005; Salter, Michaud, \& Larouche, 2010) and homecare teleassistive mobile robot interaction (Labonté et al., 2006; Michaud, Boissy, et al., 2010). Similarly, Satake et al. (2009) implemented an invitation service interaction scenario in which the robot offered shopping information and invited people to visit shops. The specifications of the robot's approach behavior were done by first testing a simpler approach in a mall, during which the velocity limitations of the platform in relation to engaging people were observed. Mutlu and Forlizzi (2008) eexamined how aspects of workflow, social/emotional, political, and environmental contexts influenced how hospital workers used, perceived, and interacted with a robot, while outlining interruptibility constraints that could not have 
been identified without preliminary experimentation on a real platform. Also, the design of the Snackbot (M. K. Lee et al., 2009) first involved the use of the CMAssist robot to conduct an early technology feasibility study.

Consequently, this paper reports on the design and control of IRL-0 and on its use in preliminary studies involving the integration of verbal with nonverbal modalities, e.g., facial expressions, head movement, arm gestures, and general mobility used to autonomously engage interaction with people. Inspired by the cub scout scenario described earlier, we implemented a short, voluntary, neutral (compared with engaging people to sell something, for instance), and simple interaction task, which consisted of having IRL-0 autonomously engage people in a public setting to get them to pick up a baseball cap lying nearby on the floor ( 3 to $4 \mathrm{~m}$ away). Assessments in terms of design (e.g., technology, control) and experimental procedures can then be made from these trials.

The paper is organized as follows: Section 2 situates related work regarding the design and the evaluation of autonomous interactive robots. Section 3presents IRL-0's hardware and software capabilities along with its behavior-based control architecture for autonomously engaging and interacting with people. Section 4 describes the two studies conducted with IRL-0, followed by Section 5 that discusses insights regarding design and evaluation factors derived from these HRI system studies.

\section{Related Work}

Most of the work on engagement in HRI involves the influence of combining verbal communication with nonverbal cues, such as head and gaze control (Bruce, Nourbakhsh, \& Simmons, 2002; Holroyd, Rich, Sidner, \& Ponsler, 2011; Imai, Ono, \& Ishiguro, 2003; J. K. Lee \& Breazeal, 2010; Yamaoka, Kanda, Ishiguro, \& Hagita, 2006; Yamazaki et al., 2007), facial features (Breazeal, Kidd, Thomaz, Hoffman, \& Berlin, 2005; Bruce et al., 2002; Faber et al., 2009), deictic gesture (Sidner, Lee, Kidd, Lesh, \& Rich, 2005; Holroyd et al., 2011; Yamaoka et al., 2006) and physical expression (Breazeal et al., 2005). Results suggest that such nonverbal cues are beneficial, but these studies usually involve one or two nonverbal modalities with an immobile platform in controlled conditions (except for (Bruce et al., 2002) which conducted trials in real-world settings). Mobility is certainly an important nonverbal modality to convey intention, as demonstrated in Terada, Shamoto, Mei, and Ito (2007) and Yamaji, Miyake, Yoshiike, De Silva, and Okada (2010). It has been used to characterize people's personal spaces in relation to a robot (Takayama \& Pantofaru, 2009), the approach and positioning of a robot relative to a seated human subject (Dautenhahn et al., 2006), and to generate a trajectory to initiate interaction with people in a mall (Kanda, Glas, Shiomi, \& Hagita, 2009; Satake et al., 2009). Saulnier et al. (Saulnier, 2011; Saulnier, Sharlin, \& Greenberg, 2011) eexamined how a robot can interrupt a human in a socially acceptable manner and observed if humans can interpret the motion and intent of a robot (through mobility and head movement). They used a Wizard of $\mathrm{Oz}$ approach and noticed the difficulty of performing the robot's behaviors consistently during experimentation. Autonomous mobility and control is therefore an interesting capability to work on regarding engagement in HRI.

To make the approach autonomous, integrating mobility with verbal and other nonverbal modalities requires the use of a control architecture to allow the robot to determine how, when, and what to do. While there are platforms integrating interaction modalities in a robot control architecture (Asfour et al., 2006; Azad, Asfour, \& Dillmann, 2007; Clodic et al., 2006; Michalowski et al., 2007; Michaud et al., 2007; Siegwart et al., 2003; Simmons et al., 2003; J. G. Trafton, Bugajska, Fransen, \& Ratwani, 2008; J. Trafton, Fransen, Harrison, \& Bugajska, 2009), their interaction capabilities are limited and usually illustrated in demonstrations without HRI evaluations; see Rousseau (2011) for a more detailed review. One exception is Robovie-IV (Mitsunaga et al., 2008; Mitsunaga, Miyashita, Ishiguro, Kogure, \& Hagita, 2006), which looked at what makes people accept a robot in a social setting over a six-week study in an office. However, the control architecture used seemed to 
simply be event-based for dialogue management and motion control yet had no generation of arm gestures. While this capability could be integrated into the behavior-based architecture presented with Robovie-I (Ishiguro et al., 2001) for autonomous HRI, this has yet to be demonstrated. Our contribution with the work reported in this paper is to present a complete integration of multi-modal interaction capabilities (voice, facial expressions, head movements, arm gestures, mobility by approaching people) in an autonomous robot control architecture and conduct preliminary assessment of this integration in engaging people to interact with the robot.

\section{IRL-0, an Interactive Omnidirectional Robot Platform}

Figure 1 shows IRL-0, our prototyping platform made of a mobile base, an upper body frame, and a compliant arm (total weight approximately $50 \mathrm{~kg}$ ). The mobile base consists of an omnidirectional non-holonomic platform with eight actuators (Ferland et al., 2010): four for propulsion and four for steering the wheels with each wheel being able to rotate $180^{\circ}$ around its steering axis. Propulsion is done using standard DC brushless motors equipped with optical encoders and capable of reaching 1.47 m/s. DEAs (Lauria, Michaud, et al., 2008; Lauria, Legault, et al., 2008) are used for wheel steering, providing compliance, safety, and torque control capabilities to the platform. The hardware architecture consists of distributed modules for sensing and low-level control, communicating with each other through a 1 Mbps CAN bus. A Mini-ITX computer equipped with a $2.0 \mathrm{GHz}$ Core 2 duo processor running in Linux is used on-board for the robot control architecture. A Hokuyo UTM-30LX laser range finder ( $30 \mathrm{~m}$ range, $270^{\circ}$ angle and $25 \mathrm{msec} / \mathrm{scan}$ ) is placed on the base. As a built-in safety feature, the mobile base is programmed to slow down and come to a complete stop when being between $1 \mathrm{~m}$ to $0.7 \mathrm{~m}$ away from an obstacle. Nickel-metal hydride batteries provide power to the platform for up to three hours of autonomy. A wireless gamepad can be used to remotely control the mobile base if required.

The upper body comes with two loudspeakers, one Kinect motion sensor (providing a 3D point cloud with color for a $640 \times 480$ image with an angular field of view of $57^{\circ}$ horizontally and $43^{\circ}$ vertically, from $0.6 \mathrm{~m}$ to $4 \mathrm{~m}$ and at 30 frames per second) and a robot head installed on a pan-tilt unit (PTU-D46-17 from Directed Perception, actuated using step motors: pan $\pm 128^{\circ}$, tilt $\left.[-17,+25]^{\circ}\right)$. The robot speaks French using text-to-speech software: lliaphon (Béchet, 2001) for text-to-phoneme conversion and mbrola (Dutoit, Pagel, Pierret, Bataille, \& van der Vrecken, 1996) for phoneme-to-sound conversion. Figure 2 illustrates the facial expressions that the robot can make using nine servo-motors (four for the mouth, three for the eyes and two for the eyebrows). This robot face has been used to derive a design methodology for expressing emotions (Shayganfar et al., 2012). Each emotional state can be expressed by two configurations that interchange every five seconds to make facial expressions livelier.

The compliant arm is attached to the upper body and has three serial DOFs (two in the shoulder and one in the elbow), also actuated with DEAs. The arm has a range of about $0.64 \mathrm{~m}$ and can handle a payload of about $1 \mathrm{~kg}$ at its endpoint. It can sustain impacts with humans or objects and can be controlled from low to high admittance with gravity compensation (Legault et al., 2008). The arm controller receives the desired angle for each joint and generates spline trajectories between waypoints at high admittance (to be safe if it comes in contact with a person) while ensuring that the arm does not collide with the robot. Arm gestures programmed on the robot consist of the following, as shown in Figure 3: Waving, Come Closer, and Pointing. Waving is accomplished with the upper arm almost horizontal, while for the Come Closer gesture, the upper arm is in diagonal with the ground. 


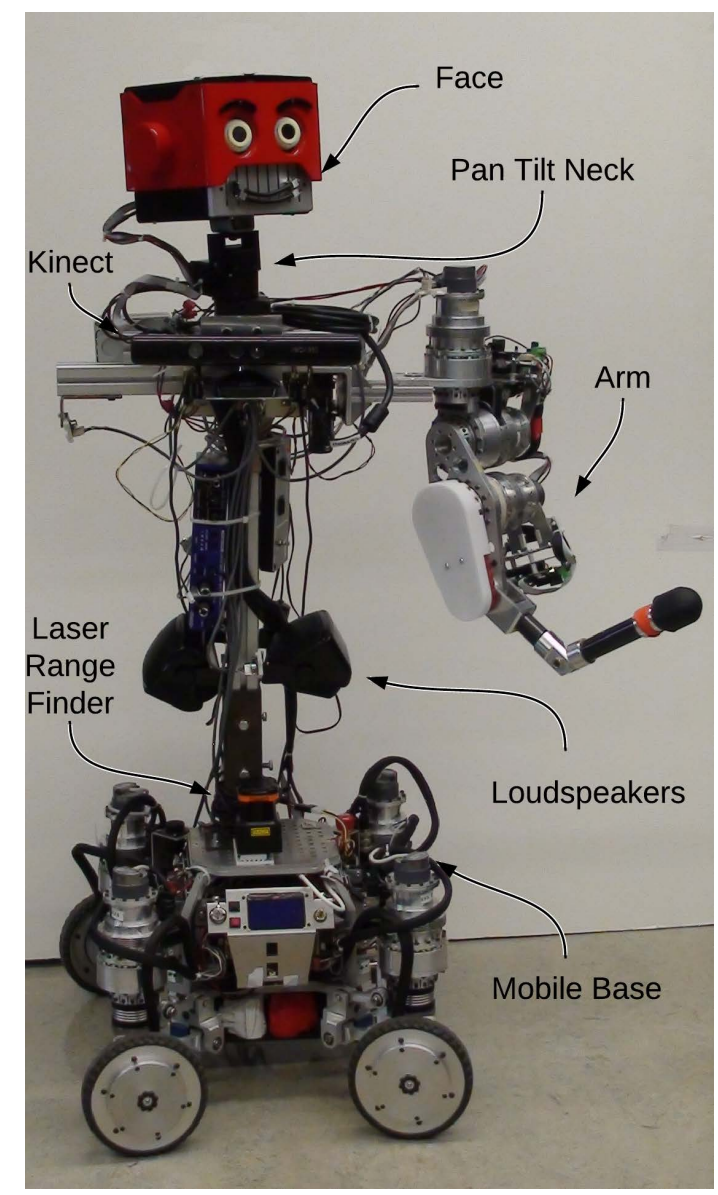

Figure 1. IRL-0

\subsection{Robot Control Architecture for Autonomously Engage People}

IRL-0's control architecture to engage interaction with people is illustrated by Figure 4, consisting of a set of behavior-producing modules (also referred to as behaviors) with priority-based arbitration. The Interaction Scenario module is a task-level executive system - implemented using SMACH, a Python library in ROS (Quigley et al., 2009) to build state machines (Bohren et al., 2011) that activate behaviors or inhibit data streams. All modules of the control architecture can access the odometry and joint states of the robot using the ROS tf package, which manages and keeps track of every coordinate frames of the robot in the world's coordinate frame. Using data from the Kinect sensor and the ROS OpenNI library, the Body Shape Detection module extracts the center of a mass of people and only tracks the first person detected until their body leaves the sensor's field of view. However, the Kinect's limited field of view makes the robot only able to try to physically intercept people walking nearby. To provide a wider detection range, the Leg Detection module uses laser range finder data to identify potential leg positions (with a reliability estimation derived from perceived leg velocity) over $180^{\circ}$ and a $5 \mathrm{~m}$ range, based on the approach presented in Arras, Grzonka, Luber, and Burgard (2008). Our implementation requires up to one second to validate the 


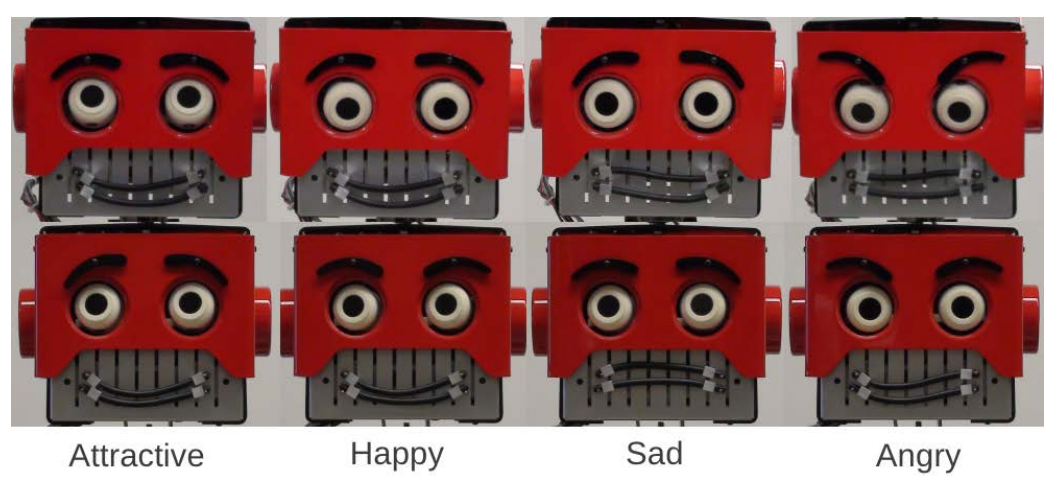

Figure 2. IRL-0's facial expressions

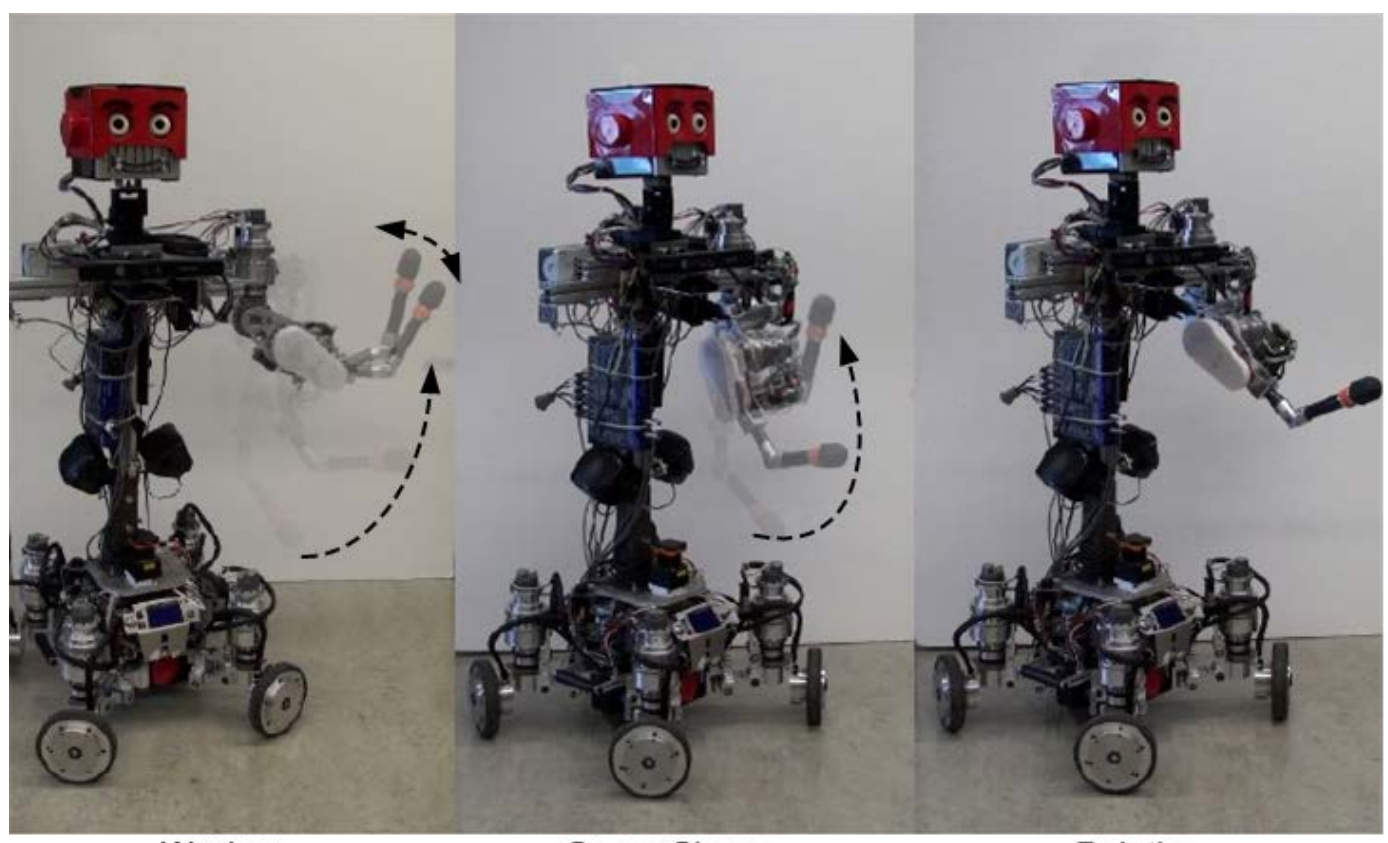

Waving

Come Closer

Pointing

Figure 3. IRL-0's arm gestures

presence of a person using leg detection. Position and direction of the first pair of legs detected are provided. Combining leg detection with body shape detection allows the robot to first identify potential targets over a large field of view using the laser range finder and then concentrate on the Kinect data, which are more reliable for tracking people.

Seven behaviors are used to control the robot's actions. These behaviors are described below, listed from the lowest to the highest priority:

- Meet Person. When the legs of a person are detected, the Waving gesture is initiated with the associated voice message and makes the robot move toward the person. If the legs are detected on 


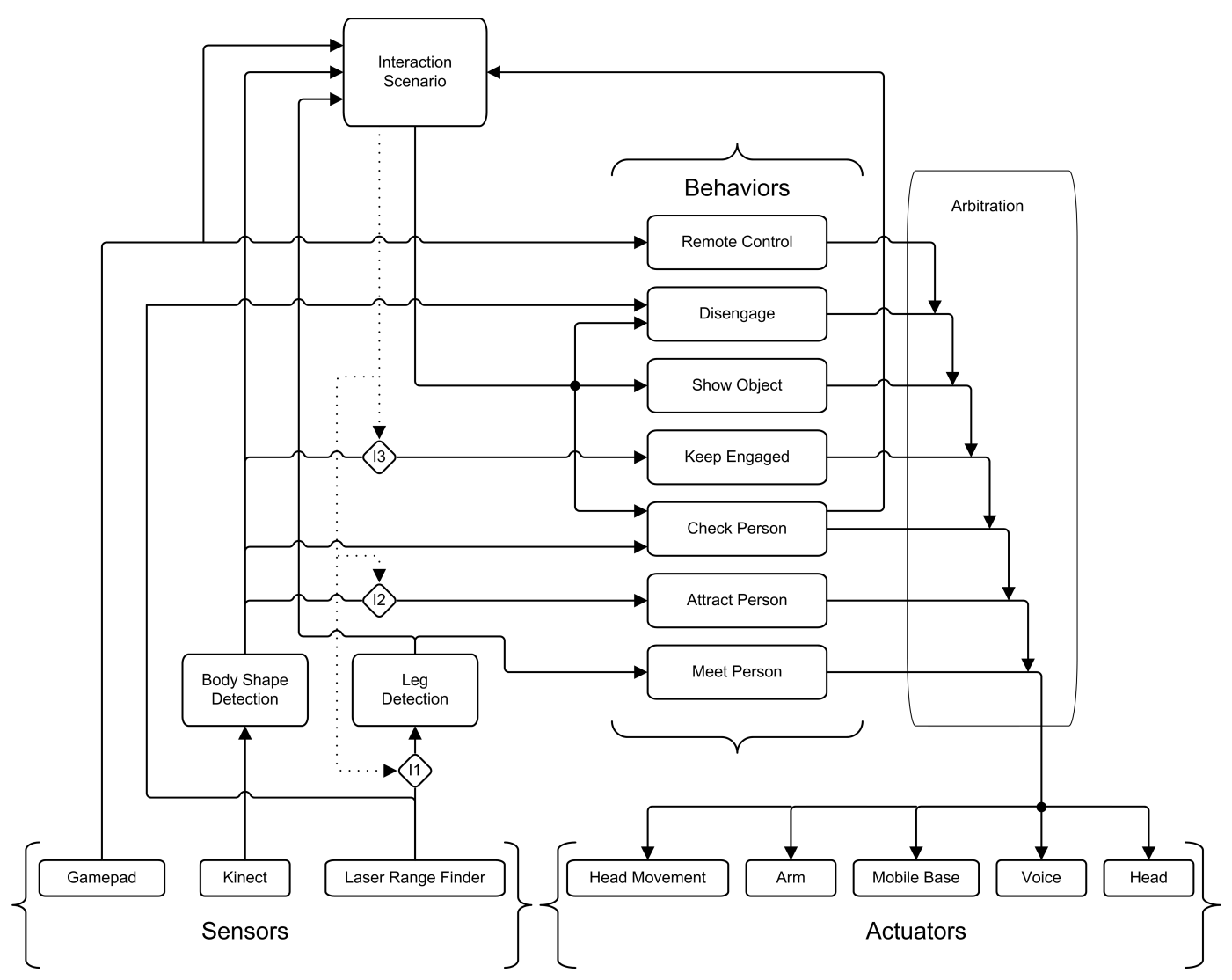

Figure 4. IRL-0's control architecture

either side of the robot, the behavior generates a curved trajectory $(4 \mathrm{~m}$ radius trajectory at $0.6 \mathrm{~m} / \mathrm{s}$ for 3 seconds with trapezoidal acceleration/deceleration profiles over $1.2 \mathrm{~s}-$ see Figure 6) with the upper body oriented perpendicular to it. Dautenhahn et al. (2006) reports that $0.4 \mathrm{~m} / \mathrm{s}$ was found to be either too slow or about right for a robot moving toward a seated person. Since IRL-0 has to move faster to intercept moving people, we set the limit to $0.6 \mathrm{~m} / \mathrm{s}$ for safe and secure approaches. No trajectory is generated if the legs are located in front $\left([-45,+45]^{\circ}\right)$ of the robot.

- Attract Person. A body shape perceived using the Kinect, allows the robot to maintain a socially acceptable distance (from 1 to $2 \mathrm{~m}$ ) and keeps the robot facing the person. This distance was set to comply with the upper end of personal distance $(0.45 \mathrm{~m}$ to $1.2 \mathrm{~m})$ and the lower end of social distance $(1.2 \mathrm{~m}$ to $3 \mathrm{~m}$ ) (Hall, 1966). Velocity commands are limited to $0.5 \mathrm{rad} / \mathrm{s}$ and $0.3 \mathrm{~m} / \mathrm{s}$.

- Check Person. This behavior checks whether the person executes the robot's request by going toward the location of the object involved in the interaction scenario. Rotation commands may be sent to the mobile base to keep track of the person (using the Body Shape Detection data). Velocity commands are limited to $0.5 \mathrm{rad} / \mathrm{s}$ and $0.3 \mathrm{~m} / \mathrm{s}$. The behavior sends an indication to the Interaction Scenario module when the person is detected in a $1 \mathrm{~m} \times 0.8 \mathrm{~m}$ range around the baseball cap.

- Keep Engaged. Using body shape position provided by the Kinect, this behavior tracks the person by rotating the mobile base (maximum $1 \mathrm{rad} / \mathrm{s}$ ) and the pan-tilt unit. If the person is located $[-5,+5]^{\circ}$ in front of the robot, only the pan-tilt unit is used, positioning it to follow the center of 


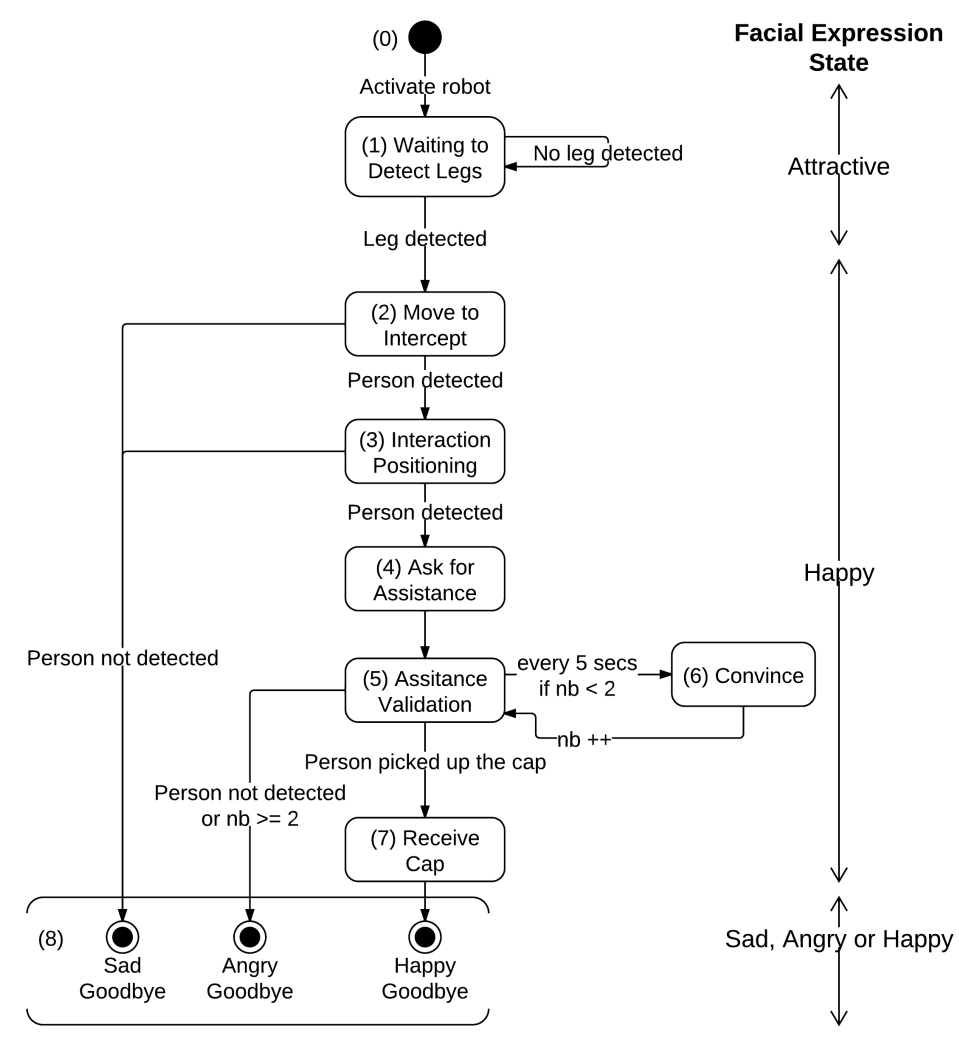

Figure 5. Finite-state machine of the Interaction Scenario module

the face (an estimated $1 \mathrm{~m}$ above the center of mass of the person). Otherwise, the behavior makes the robot rotate toward the person's position to assist tracking done by the pan-tilt unit.

- Show Object. This behavior makes the robot point toward the position of the object used in the interaction scenario (i.e., the baseball cap). It does so by generating voice commands, performing the Pointing gesture, and orienting IRL-0's mobile base (rotated by an angle bisector to the angle between the person and the object) and the head toward the object.

- Disengage. While generating vocal messages and making the Waving gesture, this behavior makes the robot base return to the position it was in when engaging interaction. The trajectory is generated using ROS local navigation planner and laser range finder data.

- Remote Control. The gamepad allows the experimenter to reposition the mobile base or to stop the robot for security purposes only if necessary. It can also be used to directly demonstrate the robot's capabilities.

The Interaction Scenario module activates Check Person, Show Object, and Disengage behaviors by specifying a specific goal to achieve: Check Person and Show Object get activated when they receive the position of the object to monitor, while Disengage is activated when it receives a position to go to. 


\subsection{Interaction Scenario}

Figure 5 illustrates the finite-state machine implemented in the Interaction Scenario module to manage how the robot interacts with people in this context, with Table 1 presenting the dialogue generated by the robot for the different states. The right side of Figure 5 indicates which facial expressions the robot adopts according to the states of the interaction scenario. These states are:

(0) This is an idle state during which only the Remote Control behavior is activated. Using the wireless gamepad, the experimenter can position the robot and activate autonomous interaction when everything is set to begin an experiment.

(1) Waiting to Detect Legs. In this state, data is allowed to be processed by the Leg Detection module, in search of a potential person for initiating interaction.

(2) Move to Intercept. Once the legs of a person are detected and tracked, the Meet Person behavior is used until the Body Shape Detection returns a valid detection of a person, which means that the person is close by and in front of the robot. Data from the laser range finder are then inhibited (box I1) to minimize computational load, and body detection data are allowed (box I2) to be transmitted to the Attract Person behavior.

(3) Interaction Positioning. The Attract Person tries to keep a socially acceptable distance between the robot and the person (as described earlier in this section for this behavior). Figure 6 illustrates the trajectory generated going from state (0) to state (3).

(4) Ask for Assistance. If the person is being located using the Kinect, the Show Object (making the robot first try to direct the attention of the person toward the baseball cap) and Keep Engaged (triggered using box I3, to turn back toward the person to keep the person engaged in the interaction) behaviors are activated.

(5) Assistance Validation. In this state, the robot validates whether the person is responding to its request for assistance. Keep Engaged tries to keep visual contact with the person, and Check Person behavior checks whether the person has been detected in a $1 \mathrm{~m} \times 0.8$ $\mathrm{m}$ range around the baseball cap. If so, the Interaction Scenario module considers that the person has carried out the robot's request.

(6) Convince. With Keep Engaged and Check Person activated, if the person is still being detected but does not go take the baseball cap, the robot vocally tries to convince the person to do so through specific vocal requests.

(7) Receive Cap. In this state, the Keep Engaged behavior is used to keep the person in the robot's field of view. Note that the robot does not detect that it receives the baseball cap: it considers that the person has given the baseball cap back after a certain time ( 3 sec., fixed empirically).

(8) Goodbye. Depending on how ends the interaction with the person, vocal messages are generated and the Disengage behavior is activated to make the robot go back to its original position.

The robot control architecture modules were designed by implementing each interaction modality individually, then by integrating them into behaviors and the Interaction Scenario module (see Section 3.2), and finally by successfully testing them (unit and system testing) in controlled laboratory conditions. Timing and parameters were finally adjusted during preliminary trials conducted in real-world settings. Table 2 shows the theoretical minimum and maximum amount of time each state can take. Total interaction length ranges from 27 to $50 \mathrm{sec}$. 


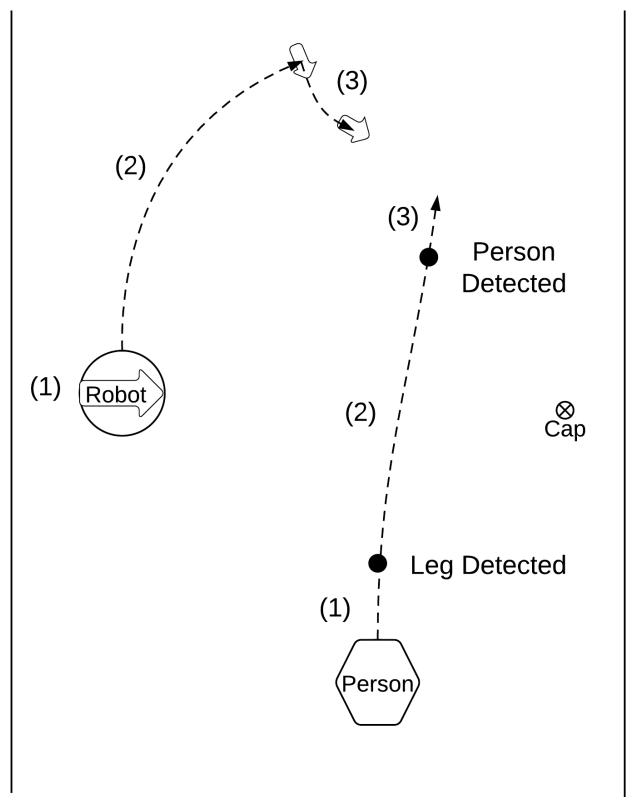

Figure 6. IRL-0's trajectory when approaching a person

Table 1: Dialogues used for the interaction scenario

ID Dialogue in French (English)

(2) Bonjour, je voudrais te parler. (Hi! I would like to talk to you.)

(3) J'ai quelque chose à te demander. (I have something to ask you.)

(4) Peux-tu me rapporter ma casquette, s'il te plaît? (Can you bring me back my cap, please?)

(6) Allez, fais un effort! (Come on, make an effort!)

(6) Tu es sûr que tu ne veux pas m'aider. (Are you sure you don't want to help me?)

(6) Cela me ferait plaisir! (This would make me so happy!)

(7) Merci beaucoup. (Thanks a lot.)

(8) Dommage ... au revoir. (Too bad ... goodbye.)

(8) Bonne journée! (Have a nice day!) 
Table 2: State duration of the interaction scenario

\begin{tabular}{ccc}
\hline States & Min (sec.) & Max (sec.) \\
\hline$(0)$ & 0 & 0 \\
$(1)$ & 5 & 5 \\
$(2)$ & 4 & 10 \\
$(3)$ & 4 & 10 \\
$(4)$ & 3 & 3 \\
$(5)$ & 3 & 4 \\
$(6)$ & 0 & 10 \\
$(7)$ & 3 & 3 \\
$(8)$ & 5 & 5 \\
\hline Total & 27 & 50 \\
\hline
\end{tabular}

\section{HRI Evaluation with IRL-0}

IRL-0 has five interaction modalities: one verbal - Voice (V), and four nonverbal - Facial expressions (F), Head movement (H), Arm gestures (A) and Mobility (M). The interaction scenario cannot be accomplished by only using the nonverbal modalities. Because our intent is to conduct preliminary studies by simply using IRL-0 as a prototyping platform, we decided to only test four out of eight possible configurations, each configuration incrementally adding one modality to the set:

- Voice and Facial expression modalities (VF).

- Voice, Facial expression, and Head movement modalities (VFH).

- Voice, Facial expression, Head movement, and Arm gestures modalities (VFHA).

- Voice, Facial expression, Head movement, Arm gestures, and Mobility modalities (VFHAM).

The influences of facial expressions in HRI have already been studied (Breazeal et al., 2005; Bruce et al., 2002; Faber et al., 2009): it is usually what is used by interactive robots with facial expression capability, with voice being the primary modality to provide basic information about the robot's intent. Therefore, VF can be considered as the baseline configuration and VFHAM is the configuration that integrates all of the interaction capabilities of IRL-0. Each of these configurations, successfully tested and validated, can easily be selected by blocking commands to the actuators not involved in the intended configuration. The underlying objective of our studies is to examine the integration of the interaction modalities and their influences in engaging people to interact with the robot, in order to identify key issues that would influence IRL-1's design and its evaluation. Therefore, we decided to conduct a study in controlled conditions to validate the implemented interaction scenario with a group of people made aware of the interaction scenario and that IRL-0 would engage interaction with them using different set of modalities. We also conducted trials in real-world settings for uncontrolled conditions where participants passing by the robot were solicited and not made aware prior to interacting with the robot that it would try to engage interaction. In all trials, IRL-0 was positioned according to Figure 6 . The studies received ethics approval with no use of video-recording (because some trials were done in public areas).

\subsection{Study in Controlled Conditions}

The test population consists of 35 able-bodied participants ( 23 males and 12 females) who completed trials in all four interaction configurations (sequentially and random order, with about a minute pause between trials). The experimenter was located nearby without being too close as to be considered 
part of the interaction. This allowed the experimenter to observe what was going on and to intervene for security purposes if necessary. Once the trials were completed, participants were asked to rank the following elements in relation to the configurations and the modalities observed:

Q1.1 Configuration preference, from 1 (most preferred) to 4 (least preferred): providing an overall assessment of the integration of interaction modalities on IRL-0.

Q1.2 Modality usefulness in the interaction scenario: considering it to be useful, useless, or harmful to the interaction.

Participants could also provide general comments about their experience in interacting with IRL-0.

4.1.1 Configuration Preference (Q1.1) The Freidman Test (Siegel, 1988) was used as a nonparametric statistical test for ranks of observations to determine if a significant difference existed between configuration preference. Equations 1 and 2 were used to identify the significant differences between the preference of each configuration for Q1.1. Equation 1 defines the minimum difference $\Delta \bar{R}_{Q 1.1}$, with $k=4$ (the number of compared configurations), $N=35$ (the total number of participants), and $z_{\alpha / k(k-1)}$ the abscissa value from the unit normal distribution above which $\alpha / k(k-1)$ percent of the distribution lies (set according to $\alpha=0.05$, the level of the test's significance; refer to Seigel (1988), Appendix A, for more information. Equation 2 expresses that the difference of the averaged rank of preference for two compared conditions $\left(\bar{R}_{i}\right.$ and $\bar{R}_{j}, i$ and $j$ being the conditions considered) must exceed the minimum difference $\Delta \bar{R}_{c}$ to be considered to have significant differences. The average rank for each condition condition was calculated by adding every rank of the condition and dividing it by the number of participants in the condition. The resulting $\Delta \bar{R}_{c}$ value for Q1.1 was 0.81.

$$
\begin{gathered}
\Delta \bar{R}_{c}=z_{\alpha / k(k-1)} \sqrt{\frac{k(k+1)}{6 N}} \\
\left|\bar{R}_{i}-\bar{R}_{j}\right| \geq \Delta \bar{R}_{c}
\end{gathered}
$$

Box plots were used to illustrate the results by representing outliers (circle), extremes (star), the smallest observation (lowest horizontal line), the lower quartile (lower end of the box), the median (horizontal line in the box), the upper quartile (upper end of the box), and the highest observation (highest horizontal line). Figure 7 illustrates for the test population that the preferred configuration was VFHAM $\left(\bar{R}_{V F H A M}=1.81\right)$, followed by VFHA $\left(\bar{R}_{V F H A}=2.01\right)$, VFH $\left(\bar{R}_{V F H}=2.64\right)$, and finally by $\operatorname{VF}\left(\bar{R}_{V F}=3.53\right)$. Significant differences are presented in bold in Table 3 . Results suggest that participants prefered interacting with the robot when Head movement $(\mathrm{H})$, Arm gestures (A) and Mobility (M) modalities were added to Voice (V) and Facial expression (F). No significant differences were found between the addition of nonverbal modalities, except for the VFHAM configuration that was preferred to the VFH configuration.

4.1.2 Modality Usefulness (Q1.2) Table 4 summarizes the results obtained for Q1.2 and provides more insights regarding the contributions of the modalities to the interaction. Results suggest that:

- Voice was found to be useful by all $(100 \%)$. Some participants commented that the robot's voice was clear and comprehensive, suggesting that no improvement is required for this particular modality.

- Arm gestures came in second (77\%), with $14 \%$ judging that the modality is useless and $9 \%$ finding it inappropriate. The interaction was found by some to be more user-friendly when the robot was gesturing and pointing the baseball cap, but for others some arm gestures (i.e., Waving and Come Closer) were difficult to understand. 


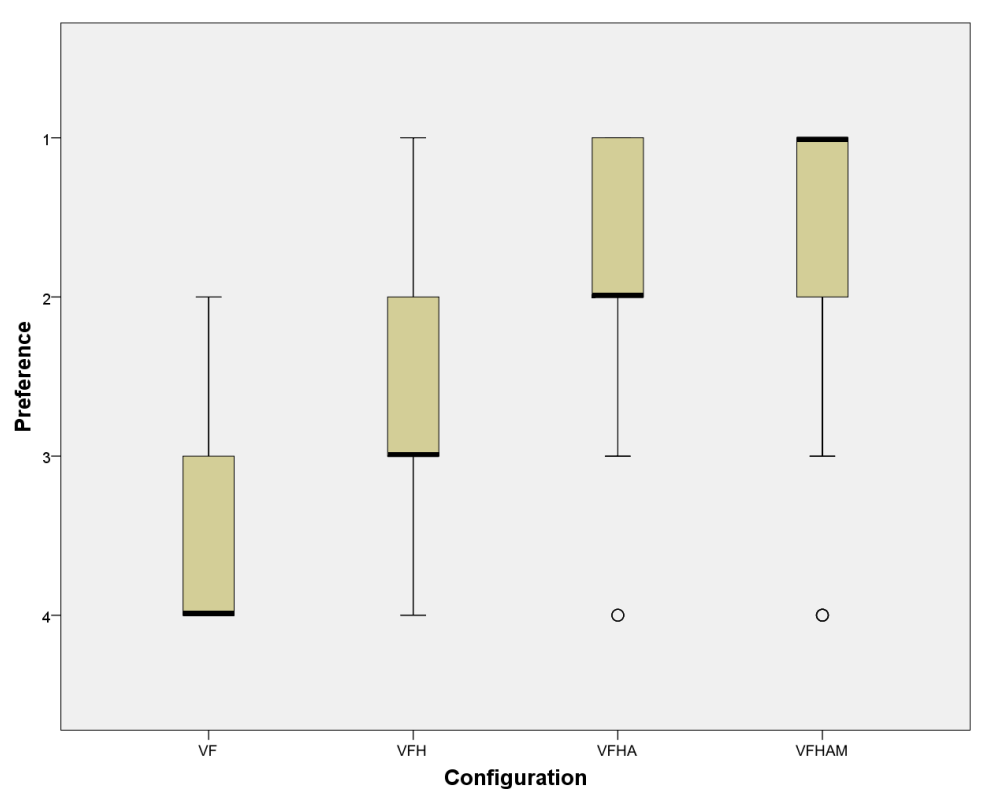

Figure 7. Q1.1 preferences for the test population

Table 3: Difference $\left(\left|\bar{R}_{i}-\bar{R}_{j}\right|\right)$ of average ranks of preferences between each configuration (Q1.1), with $\Delta \bar{R}_{c}$ $=0.81$

\begin{tabular}{llll}
\hline & $\bar{R}_{V F}$ & $\bar{R}_{V F H}$ & $\bar{R}_{V F H A}$ \\
\hline $\bar{R}_{V F H}$ & $\mathbf{0 . 8 9}$ & - & - \\
$\bar{R}_{V F H A}$ & $\mathbf{1 . 5 1}$ & 0.63 & - \\
$\bar{R}_{V F H A M}$ & $\mathbf{1 . 7 1}$ & $\mathbf{0 . 8 3}$ & 0.20 \\
\hline
\end{tabular}

- Head movement was considered useless by $31 \%$ of the participants but only $3 \%$ found it unsuitable. Some participants indicated that when the head was sitting still, they did not feel that they were engaged in a conversation with the robot. One participant indicated that the pan-tilt unit was too slow and noisy.

- Mobility was considered useful by $60 \%$ but useless by $17 \%$ and unsuitable by $23 \%$. Three participants even felt uncomfortable as the robot moved toward them. This suggests that the trajectory made by the robot when approaching a person should be revised and improved.

- Facial expressions were found useful by about half the participants (indicating that facial expressions are lovely and explicit), while the other half found them useless in relation to the interaction scenario. This observation is consistent with the fact that facial expressions are the least informative of the nonverbal modalities regarding the robot's intent for the interaction scenario.

\subsection{Study in Real-World Settings}

The objective of this study was to observe interaction with IRL-0 'in the wild', trying to engage people as they passed by in a corridor. These trials were therefore conducted in the most natural 
Table 4: Q1.2 results

\begin{tabular}{lccc}
\hline & Useful & Useless & Unsuitable \\
\hline Voice & $100 \%$ & $0 \%$ & $0 \%$ \\
Arm gestures & $77 \%$ & $14 \%$ & $9 \%$ \\
Head movement & $66 \%$ & $31 \%$ & $3 \%$ \\
Mobility & $60 \%$ & $17 \%$ & $23 \%$ \\
Facial expression & $49 \%$ & $51 \%$ & $0 \%$ \\
\hline
\end{tabular}

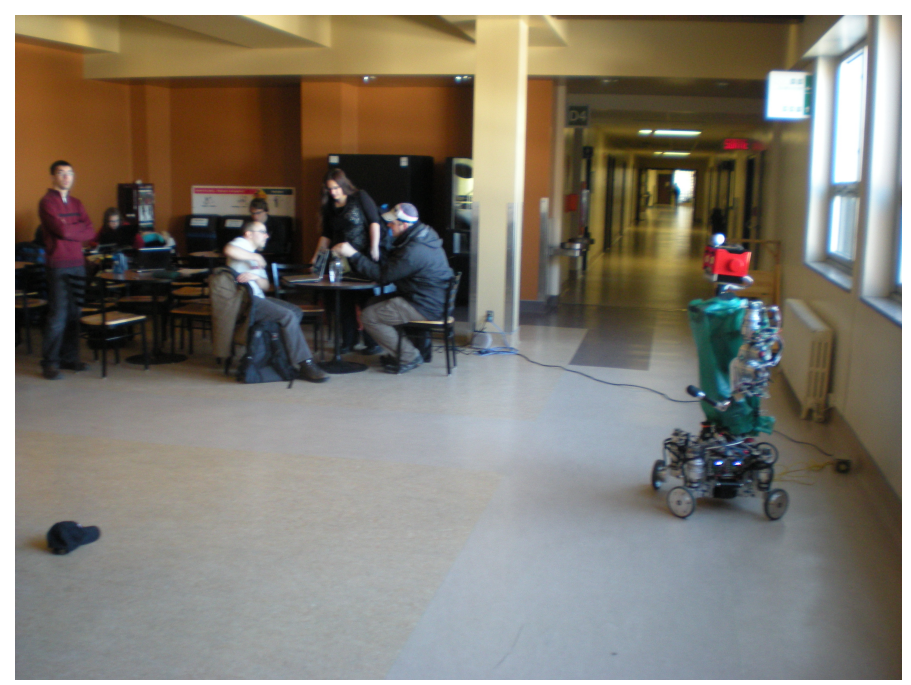

Figure 8. IRL-0 in position to interact with people in real-world settings using the VF configuration (with the robot being connected to its charger).

settings but also in the most difficult conditions for an autonomous robot. With no past experiences on what to expect, this study provides preliminary insights about the robot's capabilities, people's response, and the experimental conditions.

This study was conducted over a two-week period in eight similar locations on our university campus, like the one shown in Figure 8. According to Scopelliti, Giuliani, and Fornara (2005), a test population mainly consisting of students is probably more receptive to robots and will react more positively to it in comparison with other populations (e.g., elderly people). Therefore, our results do not represent the perception of a more generalized population; however, our study and trials within were sufficient for the assessments we wanted to make in terms of design, technology, control, and experimental procedures.

To try to minimize the novelty factor that a robot may have when placed in a public space, we demonstrated IRL-0's capabilities (controlling the robot using the gamepad) at these locations during the first week. We then experimented with the interaction scenario (autonomously carried out by the robot) in the second week. Once at a particular location, IRL-0 was set to interact with people using only one of the four configurations (VF, VFH, VFHA, VFHAM) for two to three hours. Overall, each configuration was used for about nine to ten hours. 
Two experimenters were involved in these trials. They remained about 5 to $10 \mathrm{~m}$ away from the robot to intervene if needed and to be able to observe without taking part in the interaction. One experimenter manually coded participants' reactions as they interacted with the robot (e.g., started to look at the robot, looked at the object shown, nodded to the robot, spoke, came closer, moved away, stopped, or gave back the baseball cap). The robot also recorded the duration of the interaction, the state at which participants left the interaction (to determine whether the interaction followed the scenario), and if the participant fulfilled the robot's request for assistance. Participants were then stopped by the second experimenter to see whether they would agree to fill out a short questionnaire.

Overall, IRL-0 tried 1,376 times to engage interaction (steps 1 and 2 of Figure 5) with people passing by. During these attempts, 381 trials (or 27.6\%) occurred with the robot asking for assistance (state 4 of the Interaction Scenario in Section 3.2), which is similar and slightly higher than the 22\% reported in Bruce et al. (2002) for people stopping to interact with a robot in a museum. Many people did not slow down to let the robot process the perceived information fast enough, leaving IRL-0's field of view before it could ask for assistance. This made IRL-0 either go back to its initial position or continue toward a new interaction with another person detected in its field of view.

For these 381 trials:

- 279 failed because of IRL-0's limitations in detecting or recognizing people in real-world and dynamic settings (e.g., one person would engage interaction and was replaced by another one passing in front of the robot; one person would interact with the robot while another grabbed the baseball cap).

- In the remaining 102 trials, IRL-0 was able to complete the interaction scenario with the participants (observing on average 5 reactions made by people while interacting with the robot; interaction lasted on average $29 \mathrm{sec}$ ) and 99 participants accepted our request to fill out the following questionnaire:

Q2.1 Is this your first encounter with the robot?

Q2.2 From 1 (low) to 5 (high), how well did you understand what the robot asked you?

Q2.3 From 1 (low) to 5 (high), did you like the robot?

Q2.4 Select the three primary aspects over the following six that made you stop to interact with the robot - Appearance, Attitude (global behavior), Voice, Facial expressions, Head movement, Arm gestures, or Mobility?

We found no significant difference in the data collected and the different configurations for Q2.1 to Q2.3, so no observations could be made between the configurations. Therefore, Table 5 only summarizes the results observed over the four configurations for the 99 trials with participants who filled out the questionnaire. Results for Q2.1 indicate that more than half of the participants had already seen the robot. Participants rated the robot as very understandable (Q2.2) and appreciated the robot $(\mathrm{Q} 2.3)$.

Table 6 summarizes what made participants stop to interact with IRL-0 (Q2.4). The percentages were calculated using the number of times the modality was chosen by participants and then divided by the number of valid trials for each configuration. In general, most participants were surprised and excited to interact with a robot (which was unusual for about half of them), so they liked whatever the robot did. Some participants indicated that curiosity was the main factor that made them interact with IRL-0. Because participants interacted with IRL-0 using only one set of modalities, we expected that participants interacting with IRL-0 using the VF or VFH configurations would not indicate that Arm gestures made them stop to interact with the robot - unfortunately, some did. The same happened with Mobility while using the VF or VFH configurations (IRL-0 is immobile in these conditions). 
Table 5: Results for Q2.1, Q2.2, and Q2.3

\begin{tabular}{lccccc}
\hline & Q2.1 & Q2.2 & Q2.3 & $\begin{array}{c}\text { Number of } \\
\text { reactions }\end{array}$ & $\begin{array}{c}\text { Interaction time } \\
(\mathrm{sec})\end{array}$ \\
\hline Mean & $54 \%$ & 4.6 & 4.0 & 5.0 & 28.8 \\
Std. Err. & $5 \%$ & 0.1 & 0.1 & 0.2 & 0.4 \\
\hline
\end{tabular}

This suggests that clarification to the question is required. Nevertheless, adding Arm gestures and especially Mobility to IRL-0's interaction modalities seems to play a useful role in engaging people to interact with the robot, and this should be an interesting avenue to explore for future studies with IRL-1.

Table 6: Results for Q2.4

\begin{tabular}{lcccccc}
\hline & Voice & Appearance & Facial expressions & Attitude & Arm gestures & Mobility \\
\hline VF & $78 \%$ & $39 \%$ & $65 \%$ & $22 \%$ & $13 \%$ & $4 \%$ \\
VFH & $63 \%$ & $60 \%$ & $50 \%$ & $40 \%$ & $13 \%$ & $10 \%$ \\
VFHA & $78 \%$ & $52 \%$ & $52 \%$ & $39 \%$ & $22 \%$ & $0 \%$ \\
VFHAM & $78 \%$ & $52 \%$ & $39 \%$ & $22 \%$ & $30 \%$ & $43 \%$ \\
\hline
\end{tabular}

\section{Discussion}

By using IRL-0 as a prototyping platform to conduct preliminary trials, our objective was to go beyond system testing in laboratory conditions to seek insights about the design (e.g., technology, control) and the experimental procedures for an autonomous robot engaging people to interact. The trials conducted demonstrate that IRL-0 can autonomously engage people according to the intended interaction scenario. No accident occurred, and the platform revealed to be robust and operating well. Verbal requests were comprehensible, people liked the robot, and the robot was coherent in its actions.

In terms of design, the following considerations can be made from the observations presented in Section 4:

- Mobility. On the one hand, motion should be made less threatening as the robot approaches people (see Section 4.1.2). On the other hand, in terms of velocity, the robot may need to move faster to catch up with people moving by. Establishing a similar compromise is reported in Satake et al. (2009), which led to the design of an anticipation model and a path planning strategy to intercept people in a mall. Slowing down and smoothly changing the trajectory using the omnidirectional capability of the platform (as the robot approaches people) should therefore be examined.

- Perception and tracking. To improve the robot's reaction time and minimize inappropriate responses (as in the 279 interrupted interactions in real-world settings) from the robot, people perception and tracking should be improved, both in terms of speed and accuracy. The robot should also be able to track the object involved in the interaction. In addition, participants were frequently observed responding back vocally to IRL- 0 . Since IRL-0 interacted vocally with people, they expected the robot to be able to understand what they were saying. This justifies the integration of the 
sound source localization, tracking, and separation system (Valin et al., 2007) for IRL-1's speech recognition capabilities.

- Arm gestures and Head movement. Improving these modalities should help make them more distinctive during the interaction (see Section 4.1.2). The use of two 4-DOFs arms with manipulators on IRL-1 will surely help. Reducing the noise generated by the pan-tilt unit for the head and improving the response time of facial expressions should be useful.

In terms of experimental procedures, one general observation from the preliminary studies conducted is the difficulty in getting statistically significant observations. Such situations are not uncommon with platforms that integrate many functionalities and are used in real-world settings, such as those seen in Saulnier (2011) and Yamaji et al. (2010). Nevertheless, these trials reveal important elements that must be taken into consideration for the completion of IRL-1 and upcoming studies.

First, letting people become familiar with the robot is a necessary step for obtaining more consistent results. The test population for the study in controlled conditions (Section 4.1) was made up of people familiar with mobile robots ( 14 male, 1 female) and people who never interacted with a mobile robot before ( 9 male, 11 female). Looking more closely at the distinctive results, no significant differences were found between the two groups but overall unfamiliar people had more scattered preferences regarding the configurations and interaction modalities. Whether trials are in controlled or in real-world settings, it may be appropriate to give participants some time to get familiarized with the robot and its capabilities in order to move past the curiosity and novelty factors when people first encounter a robot. In real-world settings, it may be preferable to let the robot interact with people at the experimental location over longer periods of time and only use data from volunteers that are familiar with the robot. Studying how familiarization with the robot impacts engagement could certainly be an interesting topic.

Second, the experimental protocol to put in place to evaluate engagement with IRL-1 should consist of a three-step process:

1. Distinct usability evaluation of nonverbal modalities in controlled conditions. The objective would be to evaluate if and by how much each modality (e.g., head movement, arm gestures, approach trajectory) is found suitable and useful. Having no significant differences between many configurations and conditions may suggest that the influences of each modality do not clearly stand out in relation to the interaction scenario. Distinct usability evaluations make it possible to identify what each modality can bring as positive and clear influences to the interaction. Five to six participants may be sufficient for conducting these evaluations (Turner, Lewis, \& Nielsen, 2006). To help observe significant differences in the different configurations to be experimented with, these evaluations may lead to changes in the implementation of the interaction scenario, in test configurations and in conditions to evaluate.

2. Evaluation of integrated nonverbal modalities in controlled conditions. Combinations of the modalities found suitable and informative in Step 1 should be examined in relation to the interaction scenario. Having participants interact with the robot in all configurations can be used for comparative analyses. Participants could indicate in what ways different sets of nonverbal modalities contribute to the interaction (e.g., making it engaging, informative, lively, and understandable). Timing measurements should also be derived to characterize the interaction dynamics that can result from the different configurations. Changes to the robot control architecture or to the interaction scenario may then be required to adapt to limitations that the robot may have.

3. Evaluate the interaction scenario in real-world settings. Two configurations should be used by two distinct groups of participants: one with the minimal set of interaction modalities required to implement the interaction scenario and another that uses the set found to be most appropriate according to findings of Step 2. Evaluation should be oriented toward the interaction scenario through quantitative measures (e.g., success or failure to engage people, reaction time to the request made 
by the robot, and task completion), a questionnaire (e.g., evaluate the perceived integrated influences of the modalities on the interaction) and qualitative observations (e.g., verbal comments and observations made as people interact with the robot).

Following this process will make it possible to identify what can lead to more significant observations between the interaction modalities and their integration, guide how and what to experiment with real-world conditions, and describe what to observe.

\section{Conclusion and Future Work}

The design and the evaluation of interactive autonomous robots involve addressing, at the system level, complex engineering, programming, reasoning, and human factor study issues. Many factors influenced by the robot's hardware, software, capabilities, tasks, and operating conditions must be taken into consideration for determining the experimental methodology. The paper provides a complete description of IRL-0's autonomous control architecture integrating verbal and non-verbal modalities to interact with people in real-world settings. Using IRL-0, we completed a full integrated design and evaluation cycle of interaction modalities in controlled and real-world settings. It demonstrates that IRL-0 can autonomously engage people by using its modalities appropriately and within their limits, pointing out improvements to be made to the approach trajectory, for people perception, tracking and vocal interaction, and for arm gestures and head movements. These preliminary studies also provide important insights on what to focus on and how to proceed with the experimental design and evaluation of an interactive autonomous robot engaging people to interact. In the absence of formal or empirical methods to support the design of interactive autonomous robots, we believe that the approach presented could provide interesting guidelines beneficial for the development of advanced platforms. IRL-0 is now dismantled, and IRL-1 is being prepared for upcoming HRI system studies according to the design and the evaluation considerations outlined in this paper. Data gathered with IRL-0 will be useful for comparative evaluations with IRL-1, allowing further evaluations of the impacts of these considerations.

\section{Acknowledgements}

This work was supported in part by the Natural Sciences and Engineering Research Council of Canada, the Canadian Foundation for Innovation, and the Canada Research Chair program. The authors want to thank David Brodeur and all the volunteers who took part in the trials.

\section{References}

Arras, K. O., Grzonka, S., Luber, M., \& Burgard, W. (2008). Efficient people tracking in laser range data using a multi-hypothesis leg-tracker with adaptive occlusion probabilities. In Proceedings from the IEEE International Conference on Robotics and Automation (pp. 1710-1715). doi:10.1109/ROBOT.2008.4543447.

Asfour, T., Regenstein, K., Azad, P., Schroder, J., Bierbaum, A., Vahrenkamp, N., \& Dillmann, R. (2006). ARMAR-III: An integrated humanoid platform for sensory-motor control. In Proceedings from 6th IEEE-RAS International Conference on Humanoid Robots (p. 169-175). doi:10.1109/ICHR.2006.321380.

Azad, P., Asfour, T., \& Dillmann, R. (2007). Stereo-based 6D object localization for grasping with humanoid robot systems. In Proceedings from the IEEE/RSJ International Conference on Intelligent Robots and Systems (p. 919-924). doi:10.1109/IROS.2007.4399135.

Béchet, F. (2001). Lia_phon : Un système complet de phonétisation de textes. Traitement Automatique des Langues, 42(1), 47-67.

Bohren, J., Rusu, R. B., Jones, E. G., Marder-Eppstein, E., Pantofaru, C., Wise, M., ... Holzer, S. (2011). Towards autonomous robotic butlers: Lessons learned with the PR2. In Proceedings from the IEEE International Conference on Robotics and Automation. doi:10.1109/ICRA.2011.5980058. 
Borst, C., Wimbrock, T., Schmidt, F., Fuchs, M., Brunner, B., Zacharias, F., .. Hirzinger, G. (2009). Rollin' Justin - Mobile platform with variable base. In Proceedings from the IEEE International Conference on Robotics and Automation (p. 1597-1598). doi:10.1109/ROBOT.2009.5152586.

Breazeal, C., Kidd, C., Thomaz, A., Hoffman, G., \& Berlin, M. (2005). Effects of nonverbal communication on efficiency and robustness in human-robot teamwork. In Proceedings from the IEEE/RSJ International Conference on Intelligent Robots and Systems (p. 708 - 713). doi:10.1109/IROS.2005.1545011.

Bruce, A., Nourbakhsh, I., \& Simmons, R. (2002). The role of expressiveness and attention in human-robot interaction. In Proceedings from the IEEE International Conference on Robotics and Automation (pp. 4138-4142). doi:10.1109/ROBOT.2002.1014396.

Chen, T. L., \& Kemp, C. (2011). A direct physical interface for navigation and positioning of a robotic nursing assistant. Advanced Robotics, 25(5), 605-627.

Clodic, A., Fleury, S., Alami, R., Chatila, R., Bailly, G., Brethes, L., .. Montreuil, V. (2006). Rackham: An interactive robot-guide. In Proceedings from 15th IEEE International Symposium on Robot and Human Interactive Communication (p. 502-509). doi:10.1109/ROMAN.2006.314378.

Dautenhahn, K., Walters, M., Woods, S., Koay, K. L., Nehaniv, C. L., Sisbot, E. A., .. Siméon, T. (2006). How may I serve you? A robot companion approaching a seated person in a helping context. In Proceedings from the ACM/IEEE International Conference on Human Robot Interaction (pp. 172-179). doi:10.1145/1121241.1121272.

Dutoit, T., Pagel, V., Pierret, N., Bataille, F., \& van der Vrecken, O. (1996). The MBROLA project: Towards a set of high quality speech synthesizers free of use for non commercial purposes. In Proceeding from the Fourth International Conference on Spoken Language Processing (pp. 1393-1396). doi:10.1109/ICSLP.1996.607874.

Faber, F., Bennewitz, M., Eppner, C., Gorog, A., Gonsior, C., Joho, D., .. Behnke, S. (2009, September). The humanoid museum tour guide Robotinho. In Proceedings from the IEEE International Symposium on Robots and Human Interactive Communications (pp. 891-896). doi:10.1109/ROMAN.2009.5326326.

Ferland, F., Clavien, L., Frémy, J., Létourneau, D., Michaud, F., \& Lauria, M. (2010). Teleoperation of AZIMUT-3, an omnidirectional non-holonomic platform with steerable wheels. In Proceedings from the IEEE/RSJ International Conference on Intelligent Robots and Systems (p. 2515 -2516). doi:10.1109/IROS.2010.5649041.

Ferland, F., Létourneau, D., Aumont, A., Frémy, J., Legault, M.-A., Lauria, M., \& Michaud, F. (2012). Natural interaction design of a humanoid robot. Journal of Human-Robot Interaction, Special Issue on HRI Perspectives and Projects from around the Globe, 1(2), 118-134.

Graf, B., Parlitz, C., \& Hagele, M. (2009). Robotic home assistant Care-O-Bot 3 product vision and innovation platform. In Proceedings from the International Conference on Human-Computer Interaction (p. 312320). doi:10.1109/ARSO.2009.5587059.

Hall, E. T. (1966). The Hidden Dimension: Man's Use of Space in Public and Private. New York: Doubleday.

Holroyd, A., Rich, C., Sidner, C., \& Ponsler, B. (2011). Generating connection events for human-robot collaboration. In Proceedings from the 6th ACM/IEEE International Conference on Human-Robot Interaction. doi:10.1109/ROMAN.2011.6005245.

Imai, M., Ono, T., \& Ishiguro, H. (2003). Physical relation and expression: Joint attention for human-robot interaction. IEEE Transactions on Industrial Electronics, 50(4), 636-643.

Ishiguro, H., Ono, T., Imai, M., Maeda, T., Kanda, T., \& Nakatsu, R. (2001). Robovie: An interactive humanoid robot. An International Journal on Industrial Robot, 28, 498-504.

Kanda, T., Glas, D., Shiomi, M., \& Hagita, N. (2009). Abstracting people's trajectories for social robots to proactively approach customers. IEEE Transactions on Robotics, 25(6), 1382-1396.

Labonté, D., Michaud, F., Boissy, P., Corriveau, H., Cloutier, R., \& Roux, M.-A. (2006). A pilot study on teleoperated mobile robots in home environments. In Proceedings from the IEEE/RSJ International Conference on Intelligent Robots and Systems. doi:10.1109/IROS.2006.282082.

Lauria, M., Legault, M.-A., Lavoie, M.-A., \& Michaud, F. (2008). Differential elastic actuator for robotic interaction tasks. In Proceedings from the IEEE International Conference on Robotics and Automation (p. 3606 -3611). doi:10.1109/ROBOT.2008.4543763.

Lauria, M., Michaud, F., Legault, M.-A., Létourneau, D., Rétornaz, P., Nadeau, I., .. Clavien, L. (2008). 
Elastic locomotion of a four steered mobile robot. In Proceedings from the IEEE/RSJ International Conference on Intelligent Robots and Systems (p. 2721 -2722). doi:10.1109/IROS.2008.4650759.

Lee, J. K., \& Breazeal, C. (2010). Human social response toward humanoid robot's head and facial features. In Proceedings from the Conference on Human Factors in Computing Systems. New York, New York, USA. doi:10.1145/1753846.1754132.

Lee, M. K., Forlizzi, J., Rybski, P. E., Crabbe, F., Chung, W., Finkle, J., .. Kiesler, S. (2009). The snackbot: Documenting the design of a robot for long-term human-robot interaction. In Proceedings from the IEEE/ACM International Conference on Human-Robot Interaction. doi:10.1145/1514095.1514100.

Legault, M.-A., Lavoie, M.-A., Cabana, F., Jacob-Goudreau, P., Letourneau, D., Michaud, F., \& Lauria, M. (2008, sept.). Admittance control of a human centered 3 DOF robotic arm using Differential Elastic Actuators. In Proceedings from the IEEE/RSJ International Conference on Intelligent Robots and Systems (p. 4143 -4144). doi:10.1109/IROS.2008.4651039.

Michalowski, M. P., Sabanovic, S., DiSalvo, C. F., Font, D. B., Hiatt, L. M., Melchior, N., \& Simmons, R. (2007). Socially distributed perception: GRACE plays social tag at AAAI 2005. Autonomous Robots, 22(4), 385-397. doi:10.1007/s10514-006-9015-6.

Michaud, F., Boissy, P., Labonté, D., Brière, S., Perrault, K., Corriveau, H., ... Lauria, M. (2010). Exploratory design and evaluation of a homecare teleassistive mobile robotic system. Mechatronics, Special Issue on Design and Control Methodology in Telerobotics, 20, 751-766. doi:10.1016/j.mechatronics.2010.01.010.

Michaud, F., Côté, C., Létourneau, D., Brosseau, Y., Valin, J. M., Beaudry, E., . . Kabanza, F. (2007). Spartacus attending the 2005 AAAI conference. Autonomous Robots, 22(4), 369-383. doi:10.1007/s10514-0069014-7.

Michaud, F., Ferland, F., Létourneau, D., Legault, M.-A., \& Lauria, M. (2010). Toward autonomous, compliant, omnidirectional humanoid robots for natural interaction in real-life settings. Paladyn Behavioral Robotic Journal, 1(1), 57-65. doi:10.2478/s13230-010-0003-3.

Michaud, F., Laplante, J., Larouche, H., Duquette, A., Caron, S., Letourneau, D., \& Masson, P. (2005). Autonomous spherical mobile robotic to study child development. IEEE Transactions on Systems, Man, and Cybernetics, 35(4), 1-10. doi:10.1109/TSMCA.2005.850596.

Mitsunaga, N., Miyashita, T., Ishiguro, H., Kogure, K., \& Hagita, N. (2006). Robovie-IV: A communication robot interacting with people daily in an office. In Proceedings from the IEEE/RSJ International Conference on Intelligent Robots and Systems (p. 5066-5072). doi:10.1109/IROS.2006.282594.

Mitsunaga, N., Miyashita, Z., Shinozawa, K., Miyashita, T., Ishiguro, H., \& Hagita, N. (2008). What makes people accept a robot in a social environment, discussion from six-week study in an office. In Proceedings from the IEEE/RSJ International Conference on Intelligent Robots and Systems (p. 3336-3343). doi:10.1109/IROS.2008.4650785.

Mutlu, B., \& Forlizzi, J. (2008). Robots in organization: The role of workflow, social, and environmental factors in human-robot interaction. In Proceedings from the 3rd ACM/IEEE International Conference on Human-Robot Interaction (pp. 287-294). doi:10.1145/1349822.1349860.

Quigley, M., Gerkey, B., Conley, K., Faust, J., Foote, T., Leibs, J., ... Ng, A. (2009). ROS: an open-source Robot Operating System. In Proceedings from the IEEE International Conference on Robotics and Automation, Workshop on Open Source Software. Retrieved from http://ai.stanford.edu/ mquigley/papers/icra2009-ros.pdf.

Rousseau, V. (2011). Intégration et évaluation de capacités interactives d'un robot humanoïde. Master's thesis, Université de Sherbrooke, Sherbrooke, QC, Canada. Master's thesis, Université de Sherbrooke, Department of Electrical Engineering and Computer Engineering.

Salter, T., Michaud, F., \& Larouche, H. (2010). How wild is wild? A taxonomy to categorize the wildness of child-robot interaction. International Journal of Social Robotics, 2(4), 405-415.

Satake, S., Kanda, T., Glas, D. F., Imai, M., Ishiguro, H., \& Hagita, N. (2009). How to approach humans? Strategies for social robots to initiate interaction. In Proceedings from the ACM/IEEE International Conference on Human-Robot Interaction (p. 109-16). doi:10.1145/1514095.1514117.

Saulnier, P. (2011). Exploring Socially Appropriate Nonverbal Robotic Interruption. Master's thesis, Calgary, $\mathrm{AB}$, Canada. Master's thesis, University of Calgary. 
Saulnier, P., Sharlin, E., \& Greenberg, S. (2011). Exploring minimal nonverbal interruption in HRI. In Proceedings from the IEEE International Workshop on Robots and Human Interactive Communications (p. 79-86). doi:10.1109/ROMAN.2011.6005257.

Scopelliti, M., Giuliani, M. V., \& Fornara, F. (2005). Robots in a domestic setting: A psychological approach. Universal Access in the Information Society, 4(2), 146-155. doi:10.1007/s10209-005-0118-1.

Shayganfar, M., Rich, C., \& Sidner, C. (2012). A design methodology for expressing emotion on robot faces. In Proceedings from the IEEE/RSJ International Conference on Intelligent Robots and Systems (p. 4577-4583). doi:10.1109/IROS.2012.6385901.

Sidner, C. L., Lee, C., Kidd, C. D., Lesh, N., \& Rich, C. (2005). Explorations in engagement for humans and robots. Journal Artificial Intelligence, 166(1-2), 140-164. doi:10.1016/j.artint.2005.03.005.

Siegel, S. (1988). Nonparametric Statistics for the Behavioral Sciences (2nd ed.). New York : McGraw-Hill.

Siegwart, R., Arras, K. O., Bouabdallah, S., Burnier, D., Froidevaux, G., Greppin, X., .. Tomatis, N. (2003). Robox at expo.02: A large scale installation of personal robots. Robotics and Autonomous Systems, Special Issue on Socially Interactive Robots, 42, 203-222. doi:10.1016/S0921-8890(02)00376-7.

Simmons, R., Goldberg, D., Goode, A., Montemerlo, M., Roy, N., Sellner, B., .. Maxwell, B. (2003). Grace: An autonomous robot for the AAAI robot challenge. AI Magazine, 24(2), 51-72.

Takayama, L., \& Pantofaru, C. (2009). Influences on proxemic behaviors in human-robot interaction. In Proceedings from the IEEE/RSJ International Conference on Intelligent Robots and Systems (pp. 54955502). doi:10.1109/IROS.2009.5354145.

Terada, K., Shamoto, T., Mei, H., \& Ito, A. (2007). Reactive movements of non-humanoid robots cause intention attribution in humans. In Proceedings from the IEEE/RSJ International Conference on Intelligent Robots and Systems. doi:10.1109/IROS.2007.4399429.

Trafton, J., Fransen, B., Harrison, A., \& Bugajska, M. (2009). An embodied model of infant gaze-following. In D. Naval Research Lab. Washington (Ed.), Technical Report.

Trafton, J. G., Bugajska, M. D., Fransen, B. R., \& Ratwani, R. M. (2008). Integrating vision and audition within a cognitive architecture to track conversations. In Proceedings from the 3rd ACM/IEEE International Conference on Human Robot Interaction (pp. 201-208). doi:10.1045/1349822.1349849.

Turner, C. W., Lewis, J. R., \& Nielsen, J. (2006). Determining usability test sample size. In W. Karwowski \& B. Raton (Eds.), International Encyclopedia of Ergonomics and Human Factors. Boca Raton, FL: CRC Press.

Valin, J.-M., Michaud, F., \& Rouat, J. (2007). Robust localization and tracking of simultaneous moving sound sources using beamforming and particle filtering. Robotics and Autonomous Systems, 55(3), 216-228. doi:10.1016/j.robot.2006.08.004.

Wyrobek, K., Berger, E., der Loos Van, H., \& Salisbury, K. (2008). Towards a personal robotics development platform: Rationale and design of an intrinsically safe personal robot. In Proceedings from the IEEE International Conference on Robotics and Automation. doi:10.1109/ROBOT.2008.4543527.

Yamaji, Y., Miyake, T., Yoshiike, Y., De Silva, P., \& Okada, M. (2010). STB: Intentional stance grounded childdependent robot. In Proceedings from the international conference on social robotics. doi:10.1007/9783-642-17248-9_8.

Yamaoka, F., Kanda, T., Ishiguro, H., \& Hagita, N. (2006). How contingent should a communication robot be? In Proceedings from the 1st ACM SIGCHI/SIGART Conference on Human-Robot Interaction (pp. 313-320). doi:10.1145/1121241.1121294.

Yamazaki, K., Kawashima, M., Kuno, Y., Akiya, N., Burdelski, M., Yamazaki, A., \& Kuzuoka, H. (2007). Prior-to-request and request behaviors within elderly day care: Implications for developing service robots for use in multiparty settings. In Proceedings from the European Conference on Computer Supported Cooperative Work (pp. 61-78). doi:10.1007/978-1-84800-031-5_4.

Authors' name and contact information: Vincent Rousseau (vincent.rousseau@usherbrooke.ca), François Ferland (francois.ferland@usherbrooke.ca), Dominic Létourneau (dominic.letourneau@usherbrooke.ca), François Michaud (francois.michaud@usherbrooke.ca contact author), Interdisciplinary Institute for Technological Innovation, Université de Sherbrooke, 3000, boul. de l'Université, Sherbrooke (QC) Canada, J1K 0A5. 